\title{
Probabilistic Framework for the Positioning Of a Vehicle in a Combined Indoor-Outdoor Scenario
}

\author{
S.Silvia Rachel ${ }^{1}$, E.Edinda Christy ${ }^{2}$, K.Mala ${ }^{3}$, Christo Ananth ${ }^{4}$ \\ P.G. Scholars, Department of M.E. Communication Systems, \\ Francis Xavier Engineering College, Tirunelveli ${ }^{1,2,3}$ \\ Associate Professor, Department of ECE,
}

Francis Xavier Engineering College, Tirunelveli ${ }^{4}$

\begin{abstract}
The development in technology has given us all sophistications but equal amounts of threats too. This has brought us an urge to bring a complete security system that monitors an object continuously. Consider a situation where a cargo vehicle carrying valuable material is moving in an area using GPS (an outdoor sensor) we can monitor it but the actual problem arises when its movement involves both indoor (with in the industry) and outdoor because GPS has its limitations in indoor environment. Hence it is essential to have an additional sensor that would enable us a continuous monitoring/tracking with out cutoff of the signal. In this paper we bring out a solution by combining Ultra wide band $(U W B)$ with GPS sensory information which eliminates the limitations of conventional tracking methods in mixed scenario(indoor and outdoor) The same method finds application in mobile robots,

monitoring a person on grounds of security, etc.

\section{INTRODUCTION}

Vehicle localization has been often addressed separately for indoor and outdoor environments. The main differences between both cases come from the different performances of the commonly employed sensors: typically, indoors sensors (laser range finders, radio beacons, etc) are more robust and provide more accurate positioning than outdoor sensors, like for example, GPS. However, in those applications in which vehicle localization has to be approached in a mixed scenario, positioning methods relying on both technologies should coexist. Furthermore, the transitions between indoor outdoor areas, where data from both type of sensors are available, should be managed coherently and exploited as a whole.
\end{abstract}


This paper describes the application of the Particle Filters as a probabilistic framework to cope with vehicle localization where UWB or GPS positioning information is available, either separately or jointly, as for example in automatic guidance of transport vehicles among industrial facilities.

Probabilistic approaches for the positioning problem are based on the estimation of a posterior probability distribution within the space of possible positions of the vehicle. They provide nearoptimal results under certain independence assumptions and a given knowledge on the initial localization. One of such mechanism is the well known Kalman filter, which forces the uncertainty to be Gaussian distributed.

Different variations have been proposed to deal with this limitation, for example multihypothesis Kalman filters and Markov Localization. Among the markovian methods, it is remarkable the Monte Carlo localization algorithms (MCL), also called Particle Filters or Condensation Algorithms, which work by representing the posterior estimation of the possible positions by a set of weighted samples, or particles. This approach exhibits the following advantages:

They have the ability to work with almost arbitrary sensor characteristics, motion dynamics, and noise distributions, even nonlinearities.

They can represent several position hypotheses simultaneously.

Computational resources are well focused, since these methods sample proportionally to the posterior distribution.
Particle filters are easy to implement.

They provide a suitable framework for the fusion of sensory information provided by different devices.

They have also some disadvantages:

Since the prediction is supported by particles, that is, by samples, a vehicle with a well-know position can loose its track because none of the generated samples is near enough to the true position.

Paradoxically, too accurate sensors cause the impoverishment of the sample space. Christo Ananth et al. [1] proposed a system about Efficient Sensor Network for Vehicle Security. Today vehicle theft rate is very high, greater challenges are coming from thieves thus tracking/ alarming systems are being deployed with an increasingly popularity .As per as security is concerned today most of the vehicles are running on the LPG so it is necessary to monitor any leakage or level of LPG in order to provide safety to passenger. Also in this fast running world everybody is in hurry so it is required to provide fully automated maintenance system to make the journey of the passenger safe, comfortable and economical. To make the system more intelligent and advanced it is required to introduce some important developments that can help to promote not only the luxurious but also safety drive to the owner. The system "Efficient Sensor Network for Vehicle Security", introduces a new trend in automobile industry. Christo Ananth et al. [2] discussed about Intelligent Sensor Network for Vehicle Maintenance System. Modern automobiles are no longer mere mechanical devices; they are pervasively monitored through various 
sensor networks \& using integrated circuits and microprocessor based design and control techniques while this transformation has driven major advancements in efficiency and safety.

In the existing system the stress was given on the safety of the vehicle, modification in the physical structure of the vehicle but the proposed system introduces essential concept in the field of automobile industry. It is an interfacing of the advanced technologies like Embedded Systems and the Automobile world. This "Intelligent Sensor Network for Vehicle Maintenance System" is best suitable for vehicle security as well as for vehicle's maintenance. Further it also supports advanced feature of GSM module interfacing. Through this concept in case of any emergency or accident the system will automatically sense and records the different parameters like LPG gas level, Engine Temperature, present speed and etc. so that at the time of investigation this parameters may play important role to find out the possible reasons of the accident.

Further, in case of accident $\&$ in case of stealing of vehicle GSM module will send SMS to the Police, insurance company as well as to the family members. Christo Ananth et al. [3] discussed about an eye blinking sensor. Nowadays heart attack patients are increasing day by day."Though it is tough to save the heart attack patients, we can increase the statistics of saving the life of patients $\&$ the life of others whom they are responsible for. The main design of this project is to track the heart attack of patients who are suffering from any attacks during driving and send them a medical need \& thereby to stop the vehicle to ensure that the persons along them are safe from accident. Here, an eye blinking sensor is used to sense the blinking of the eye. spO2 sensor checks the pulse rate of the patient. Both are connected to micro controller.If eye blinking gets stopped then the signal is sent to the controller to make an alarm through the buffer. If $\mathrm{spO} 2$ sensor senses a variation in pulse or low oxygen content in blood, it may results in heart failure and therefore the controller stops the motor of the vehicle. Then Tarang F4 transmitter is used to send the vehicle number \& the mobile number of the patient to a nearest medical station within $25 \mathrm{~km}$ for medical aid. The pulse rate monitored via LCD .

The Tarang F4 receiver receives the signal and passes through controller and the number gets displayed in the LCD screen and an alarm is produced through a buzzer as soon the signal is received. Christo Ananth et al. [4] discussed about a system, GSM based AMR has low infrastructure cost and it reduces man power. The system is fully automatic, hence the probability of error is reduced. The data is highly secured and it not only solve the problem of traditional meter reading system but also provides additional features such as power disconnection, reconnection and the concept of power management. The database stores the current month and also all the previous month data for the future use. Hence the system saves a lot amount of time and energy. Due to the power fluctuations, there might be a damage in the home appliances.

Hence to avoid such damages and to protect the appliances, the voltage 
controlling method can be implemented. Christo Ananth et al. [5] discussed about a system, in this project an automatic meter reading system is designed using GSM Technology. The embedded micro controller is interfaced with the GSM Module. This setup is fitted in home. The energy meter is attached to the micro controller. This controller reads the data from the meter output and transfers that data to GSM Module through the serial port. The embedded micro controller has the knowledge of sending message to the system through the GSM module. Another system is placed in EB office, which is the authority office. When they send "unit request" to the microcontroller which is placed in home. Then the unit value is sent to the EB office PC through GSM module. According to the readings, the authority officer will send the information about the bill to the customer. If the customer doesn't pay bill on-time, the power supply to the corresponding home power unit is cut, by sending the command through to the microcontroller. Once the payment of bill is done the power supply is given to the customer. Power management concept is introduced, in which during the restriction mode only limited amount of power supply can be used by the customer. Christo Ananth et al. [6] proposed a method in which the minimization is per-formed in a sequential manner by the fusion move algorithm that uses the QPBO min-cut algorithm. Multi-shape GCs are proven to be more beneficial than single-shape GCs.

Hence, the segmentation methods are validated by calculating statistical measures. The false positive (FP) is reduced and sensitivity and specificity improved by multiple MTANN. Christo Ananth et al. [7] proposed a system, this system has concentrated on finding a fast and interactive segmentation method for liver and tumor segmentation. In the preprocessing stage, Mean shift filter is applied to CT image process and statistical thresholding method is applied for reducing processing area with improving detections rate. In the Second stage, the liver region has been segmented using the algorithm of the proposed method. Next, the tumor region has been segmented using Geodesic Graph cut method. Results show that the proposed method is less prone to shortcutting than typical graph cut methods while being less sensitive to seed placement and better at edge localization than geodesic methods.

This leads to increased segmentation accuracy and reduced effort on the part of the user. Finally Segmented Liver and Tumor Regions were shown from the abdominal Computed Tomographic image. Christo Ananth et al. [8] proposed a system, in which a predicate is defined for measuring the evidence for a boundary between two regions using Geodesic Graphbased representation of the image. The algorithm is applied to image segmentation using two different kinds of local neighborhoods in constructing the graph. Liver and hepatic tumor segmentation can be automatically processed by the Geodesic graph-cut based method. This system has concentrated on finding a fast and interactive segmentation method for liver and tumor segmentation. In the preprocessing stage, the CT image process is 
carried over with mean shift filter and statistical thresholding method for reducing processing area with improving detections rate. Second stage is liver segmentation; the liver region has been segmented using the algorithm of the proposed method. The next stage tumor segmentation also followed the same steps.

Finally the liver and tumor regions are separately segmented from the computer tomography image. Christo Ananth et al. [9] proposed a system in which the crossdiamond search algorithm employs two diamond search patterns (a large and small) and a halfway-stop technique. It finds small motion vectors with fewer search points than the DS algorithm while maintaining similar or even better search quality. The efficient Three Step Search (E3SS) algorithm requires less computation and performs better in terms of PSNR. Modified objected block-base vector search algorithm (MOBS) fully utilizes the correlations existing in motion vectors to reduce the computations. Fast Objected - Base Efficient (FOBE) Three Step Search algorithm combines E3SS and MOBS. By combining these two existing algorithms CDS and MOBS, a new algorithm is proposed with reduced computational complexity without degradation in quality. Christo Ananth et al. [10] proposed a system in which this study presented the implementation of two fully automatic liver and tumors segmentation techniques and their comparative assessment. The described adaptive initialization method enabled fully automatic liver surface segmentation with both GVF active contour and graph-cut techniques, demonstrating the feasibility of two different approaches. The comparative assessment showed that the graph-cut method provided superior results in terms of accuracy and did not present the described main limitations related to the GVF method. The proposed image processing method will improve computerized CT-based 3-D visualizations enabling noninvasive diagnosis of hepatic tumors. The described imaging approach might be valuable also for monitoring of postoperative outcomes through CTvolumetric assessments.

Processing time is an important feature for any computer-aided diagnosis system, especially in the intra-operative phase. Christo Ananth et al. [11] proposed a system in which an automatic anatomy segmentation method is proposed which effectively combines the Active Appearance Model, Live Wire and Graph Cut (ALG) ideas to exploit their complementary strengths. It consists of three main parts: model building, initialization, and delineation. For the initialization (recognition) part, a pseudo strategy is employed and the organs are segmented slice by slice via the OAAM (Oriented Active Appearance method). The purpose of initialization is to provide rough object localization and shape constraints for a latter GC method, which will produce refined delineation. It is better to have a fast and robust method than a slow and more accurate technique for initialization. Christo Ananth et al. [12] proposed a system which uses intermediate features of maximum overlap wavelet transform (IMOWT) as a pre-processing step. The coefficients derived 
from IMOWT are subjected to 2D histogram Grouping. This method is simple, fast and unsupervised. 2D histograms are used to obtain Grouping of color image. This Grouping output gives three segmentation maps which are fused together to get the final segmented output. This method produces good segmentation results when compared to the direct application of $2 \mathrm{D}$ Histogram Grouping.

IMOWT is the efficient transform in which a set of wavelet features of the same size of various levels of resolutions and different local window sizes for different levels are used. IMOWT is efficient because of its time effectiveness, flexibility and translation invariance which are useful for good segmentation results. Christo Ananth et al. [13] proposed a system in which OWT extracts wavelet features which give a good separation of different patterns. Moreover the proposed algorithm uses morphological operators for effective segmentation. From the qualitative and quantitative results, it is concluded that our proposed method has improved segmentation quality and it is reliable, fast and can be used with reduced computational complexity than direct applications of Histogram Clustering. The main advantage of this method is the use of single parameter and also very faster. While comparing with five color spaces, segmentation scheme produces results noticeably better in RGB color space compared to all other color spaces. Christo Ananth et al. [14] presented an automatic segmentation method which effectively combines Active Contour Model, Live Wire method and Graph Cut approach (CLG).
The aim of Live wire method is to provide control to the user on segmentation process during execution. Active Contour Model provides a statistical model of object shape and appearance to a new image which are built during a training phase. In the graph cut technique, each pixel is represented as a node and the distance between those nodes is represented as edges. In graph theory, a cut is a partition of the nodes that divides the graph into two disjoint subsets. For initialization, a pseudo strategy is employed and the organs are segmented slice by slice through the OACAM (Oriented Active Contour Appearance Model). Initialization provides rough object localization and shape constraints which produce refined delineation.

This method is tested with different set of images including CT and MR images especially 3D images and produced perfect segmentation results. Christo Ananth et al. [15] proposed a work, in this work, a framework of feature distribution scheme is proposed for object matching. In this approach, information is distributed in such a way that each individual node maintains only a small amount of information about the objects seen by the network. Nevertheless, this amount is sufficient to efficiently route queries through the network without any degradation of the matching performance. Digital image processing approaches have been investigated to reconstruct a high resolution image from aliased low resolution images. The accurate registrations between low resolution images are very important to the reconstruction of a high resolution image. The proposed feature 
distribution scheme results in far lower network traffic load.

To achieve the maximum performance as with the full distribution of feature vectors, a set of requirements regarding abstraction, storage space, similarity metric and convergence has been proposed to implement this work in $\mathrm{C}++$ and QT. Christo Ananth et al. [16] discussed about an important work which presents a metal detecting robot using RF communication with wireless audio and video transmission and it is designed and implemented with Atmel 89C51 MCU in embedded system domain. The robot is moved in particular direction using switches and the images are captured along with the audio and images are watched on the television .Experimental work has been carried out carefully. The result shows that higher efficiency is indeed achieved using the embedded system. The proposed method is verified to be highly beneficial for the security purpose and industrial purpose. The mine sensor worked at a constant speed without any problem despite its extension, meeting the specification required for the mine detection sensor. It contributed to the improvement of detection rate, while enhancing the operability as evidenced by completion of all the detection work as scheduled. The tests demonstrated that the robot would not pose any performance problem for installation of the mine detection sensor. On the other hand, however, the tests also clearly indicated areas where improvement, modification, specification change and additional features to the robot are required to serve better for the intended purpose. Valuable data and hints were obtained in connection with such issues as control method with the mine detection robot tilted, merits and drawbacks of mounting the sensor, cost, handling the cable between the robot and support vehicle, maintainability, serviceability and easiness of adjustments.

These issues became identified as a result of our engineers conducting both the domestic tests and the overseas tests by themselves, and in this respect the findings were all the more practical. Christo Ananth et al. [17] discussed about Vision based Path Planning and Tracking control using Mobile Robot. This paper proposes a novel methodology for autonomous mobile robot navigation utilizing the concept of tracking control. Vision-based path planning and subsequent tracking are performed by utilizing proposed stable adaptive state feedback fuzzy tracking controllers designed using the Lyapunov theory and particleswarm-optimization (PSO)-based hybrid approaches. The objective is to design two self-adaptive fuzzy controllers, for $\mathrm{x}$ direction and y-direction movements, optimizing both its structures and free parameters, such that the designed controllers can guarantee desired stability and, simultaneously, can provide satisfactory tracking performance for the vision-based navigation of mobile robot.

The design methodology for the controllers simultaneously utilizes the global search capability of PSO and Lyapunovtheory-based local search method, thus providing a high degree of automation. Two different variants of hybrid approaches 
have been employed in this work. The proposed schemes have been implemented in both simulation and experimentations with a real robot, and the results demonstrate the usefulness of the proposed concept. Christo Ananth et al. [18] discussed about a model, a new model is designed for boundary detection and applied it to object segmentation problem in medical images. Our edge following technique incorporates a vector image model and the edge map information. The proposed technique was applied to detect the object boundaries in several types of noisy images where the illdefined edges were encountered. The proposed techniques performances on object segmentation and computation time were evaluated by comparing with the popular methods, i.e., the ACM, GVF snake models. Several synthetic noisy images were created and tested.

The method is successfully tested in different types of medical images including aortas in cardiovascular MR images, and heart in CT images. Christo Ananth et al. [19] discussed about the issue of intuitive frontal area/foundation division in still pictures is of awesome down to earth significance in picture altering. They maintain a strategic distance from the limit length predisposition of chart cut strategies and results in expanded affectability to seed situation. Another proposed technique for completely programmed handling structures is given taking into account Graph-cut and Geodesic Graph cut calculations. This paper addresses the issue of dividing liver and tumor locales from the stomach CT pictures. The absence of edge displaying in geodesic or comparable methodologies confines their capacity to exactly restrict object limits, something at which chart cut strategies by and large exceed expectations. A predicate is characterized for measuring the confirmation for a limit between two locales utilizing Geodesic Graph-based representation of the picture. The calculation is connected to picture division utilizing two various types of nearby neighborhoods in building the chart. Liver and hepatic tumor division can be naturally prepared by the Geodesic chart cut based strategy. This framework has focused on finding a quick and intuitive division strategy for liver and tumor division.

In the pre-handling stage, Mean movement channel is connected to CT picture process and factual thresholding technique is connected for diminishing preparing zone with enhancing discoveries rate. In the Second stage, the liver area has been divided utilizing the calculation of the proposed strategy. Next, the tumor district has been portioned utilizing Geodesic Graph cut strategy. Results demonstrate that the proposed strategy is less inclined to shortcutting than run of the mill diagram cut techniques while being less delicate to seed position and preferable at edge restriction over geodesic strategies. This prompts expanded division exactness and decreased exertion with respect to the client. At long last Segmented Liver and Tumor Regions were appeared from the stomach Computed Tomographic picture. Christo Ananth et al. [20] discussed about efficient content-based medical image retrieval, dignified according to the Patterns for Next generation Database 
systems (PANDA) framework for pattern representation and management. The proposed scheme use 2-D Wavelet Transform that involves block-based lowlevel feature extraction from images. An expectation-maximization algorithm is used to cluster the feature space to form higher level, semantically meaningful patterns. Then, the 2-component property of PANDA is exploited: the similarity between two clusters is estimated as a function of the similarity of both their structures and the measure components. Experiments were performed on a large set of reference radiographic images, using different kinds of features to encode the low-level image content. Through this experimentation, it is shown that the proposed scheme can be efficiently and effectively applied for medical image retrieval from large databases, providing unsupervised semantic interpretation of the results, which can be further extended by knowledge representation methodologies. In spite of these limitations, there are practical approaches, as shown further on, to overcome these problems. This paper gives:

1. An overview of the UWB and GPS sensors as positioning technologies;

2. The mathematical formulation of Particle Filters and its use for sensor combination;

3. Some simulated results of the combination of UWB and GPS readings to estimate the pose of vehicles within mixed scenarios

\section{UWB AND GPS SENSORS OVERVIEW}

Ultra-Wide Band (UWB) is a quite new technology with major advantages for wireless communications. It is based on the transmission of short pulses in the band between 3.6 and $10.1 \mathrm{GHz}$. Apart from communication, it can also be exploited for positioning, since the distance between two antennas can be accurately derived through TOF (time-of-flight). From the localization point of view, the main advantages of this system are:

-UWB signals are not affected by multipath fading.

-The signals can penetrate through objects. -It exhibits precision ranging at centimeter level.

-As the signals are of very-low power, there can be small transmitters and receivers.

On the other hand, GPS is a satellite geolocalization technique that has been widely exploited in the last years. Basically, it uses the signals received from satellites to develop a tri-lateration process. For positioning, the system uses two radio channels in the microwave band, centered at $1575.42 \mathrm{MHz}$ and $1227.60 \mathrm{MHz}$. The accuracy of GPS can be improved by the usage of differential GPS (DGPS) to achieve a resolution of tens of centimeters. The transmitted signals cannot penetrate most materials, which limits the performance of the system and makes GPS appropriated only for outdoor applications, but not for localization among buildings, dense urban environments, forests, etc.

Another difference is the type of information provided by these sensors: UWB radio devices provide range 
measurements, while the GPS system gives the position and orientation $(x, y, p h i)$ of the vehicle. For the simulations, Gaussian models have been adopted for both sensors, being the uncertainty of the UWB characterized by the standard deviation of its measurements and the one of GPS by a $3 \times 3$ covariance matrix of the $(x, y, p h i)$ coordinates. Next, the approach for combining both types of sensor data through particle filters is presented.

\section{PARTICLE FILTERS FOR SENSOR COMBINATION IN VEHICLE POSITIONING}

-The basis of the Particle Filters: Bayes filtering.

-Particular implementation of a Particle Filter Localization algorithm which copes with the combination of different sensory devices.

\section{Bayes filtering}

Bayes filters estimate a posterior probability density, called the belief, denoted $\operatorname{Bel}(x t)$ (belief of being at position $x$ at a time $t$ ), over a space of possible positions conditioned on the observation data. These filters are based on the Markov assumption, for which the past and future data are independent. Thus, the belief function will be recursively calculated as:

$-\operatorname{Bel}\left(x_{t}\right)=\eta p\left(o_{t} \mid x_{t}\right) \int p\left(x_{t} \mid x_{t-1}, a_{t-1}\right) \operatorname{Bel}\left(x_{t-1}\right) d x_{t-1}$ where

$\eta=p\left(o_{t} \mid a_{t-1}, \ldots, o_{0}\right)^{-1}$ is a normalization constant, $o_{t}$ is the sensor observation taken at time $t$, and $a_{t}$ the action executed at time $t$. Jointly with an initial probability distribution, this equation allows us to estimate future believes about the vehicle position.For the calculation of $\operatorname{Bel}(x)$ two probability densities must be known

$$
p\left(o_{t} \mid x_{t}\right) \text { and } p\left(x_{t} \mid x_{t-1}, a_{t-1}\right)
$$

The former is the observation model or sensor model, and provides the particular characteristics of each sensor; the later is the motion model and reflects the motion behavior of the vehicle.

\section{Particle filters}

Particle Filters become an efficient way of solving the Bayes Filter (1) by representing the belief function $\operatorname{Bel}(x)$ by a set of weighted samples, or particles, distributed according to: 


$$
\operatorname{Bel}(x) \approx\left\{x^{(i)}, w^{(i)}\right\}_{i=1, \ldots, m}
$$

Particles $\left(\mathrm{x}^{(\mathrm{i})}\right)$ represent the plausible positions of the vehicle following its motion model. Weights $w^{(i)}$ also called importance factors, represent the "goodness" of each particle for approaching the real belief function. This set of pairs particle-weight permits us to easily integrate information from different sensors. The position represented by the weighted mean of the particles will be assumed as the vehicle location. The Sequential Importance Sampling (SIS) Algorithm is followed for implementing the particle filter. It is divided in four stages

\section{Step 1: Prediction.}

Draw the set of $m$ particles according to the last motion action.

\section{Step 2: Update.}

Assuming the sensors are mutually independent, the weights for particles are updated as:

$$
w^{(i)}=w_{t-1}^{(i)} \cdot p\left(o_{t}^{1} \mid x_{t}^{(i)}\right) \cdot p\left(o_{t}^{2} \mid x_{t}^{(i)}\right) \cdot \ldots \cdot p\left(o_{t}^{N} \mid x_{t}^{(i)}\right)
$$

where $N$ is the number of different observations. Particularizing to our case, the available observations will be range measurements provided by UWB sensors, positions supplied by GPS, or both, and thus their respective sensor models are considered.

\section{Step 3: Normalization.}

The new weights are normalized to represent a probability distribution as:

$$
w_{t}^{(i)}=\frac{w_{x}^{(D)}}{\sum_{i=1}^{m} w_{t}^{(i)}}
$$

\section{Step 4: Resampling.}

This stage aims to avoid particle impoverishment. In our approach, resampling is implemented as a systematic method, which is executed when the number of high-weighted particles is under a given threshold. The evolution of the filter maintains a set of particles that accurately represent the vehicle pose. . Christo Ananth et al. [21] discussed about E-plane and Hplane waveguides (Microwave Engineering) applicable to image processing. Christo 


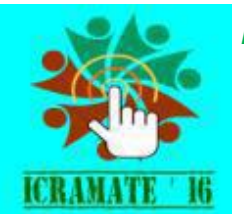

International Conference on Recent Advances in Management, Architecture, Technology and Engineering

(ICRAMATE'16)

Organized by

International Journal of Advanced Research in Management, Architecture, Technology and Engineering

(IJARMATE)

$21^{\text {st }}$ March 2016
Ananth et al. [22] discussed about principles of electronic devices to explain this application.

\section{UWB-GPS combined results}

We have setup the simulated indoor + outdoor environment depicted in Fig using 200 particles and 4 UWB beacons inside each warehouse. In this scenario, as the vehicle goes out the first warehouse, it loses UWB signals but starts to receive GPS readings. At this transition area, although the vehicle eventually only receives readings from 2 or less UWB beacons, the localization error reduced due to the measures provided by the GPS. Christo Ananth et al. [23] gave a brief outline on Electronic devices and circuits which is the basis for formation of patterns.

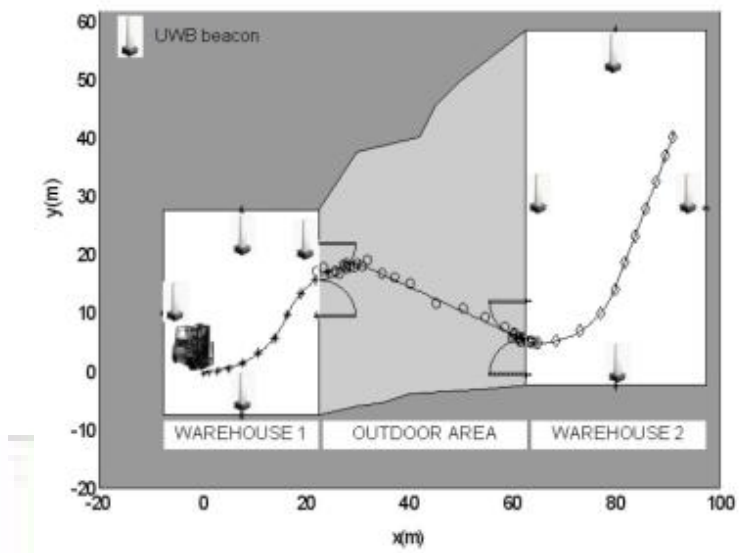

Fig.Vehicle localization within a combined indoor outdoor environment. Marks on the path indicate the estimated positions of the vehicle. Note than when both sources of information are jointly available (at transition areas), the inaccuracy of the GPS is corrected, permitting the vehicle to pass through the gates.

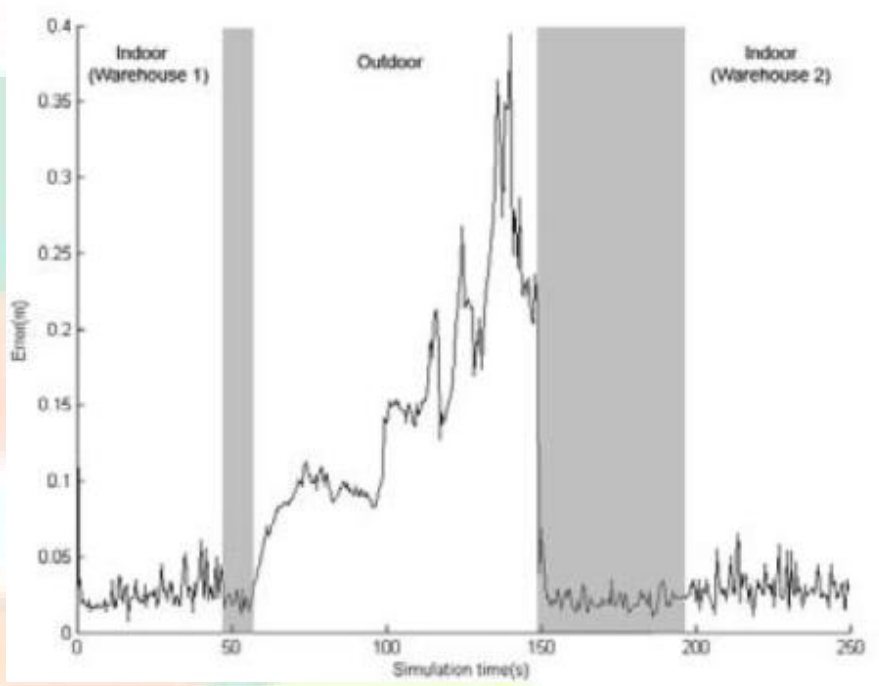

Fig.Localization error of the vehicle with respect to the ground truth path for the mixed scenario. Note the accuracy in the pose estimation along indoor as well as in mixed areas where both UWB and GPS observations are combined.

\section{CONCLUSION}

This paper describes a probabilistic framework for the positioning of a vehicle in a combined indoor + outdoor scenario. The performance of UWB sensor technology for indoor positioning and GPS for outdoor areas is studied. Simulated experiments have demonstrated the suitability of the approach .For active tracking and positioning applications, the short-pulse UWB techniques offer distinct advantages. The same approach can be exploited for extended-operation RF identification (RFID), for monitoring a VIP on grounds of security or even an accused under imprisonment during transfer from one place 


\section{to another, in mobile robots and to check smuggling of goods.} References:

[1] Christo Ananth, I.Uma Sankari, A.Vidhya, M.Vickneshwari, P.Karthiga, "Efficient Sensor Network for Vehicle Security", International Journal of Advanced Scientific and Technical Research (IJASR), Volume 2, Issue 4, March-April 2014,pp - 871877

[2] Christo Ananth, C.Sudalai@UtchiMahali, N.Ebenesar Jebadurai, S.Sankari@Saranya, T.Archana, “Intelligent sensor Network for Vehicle Maintenance system", International Journal of Emerging Trends in Engineering and Development (IJETED), Vol.3, Issue 4, May 2014, pp-361-369

[3] Christo Ananth, S.Shafiqa Shalaysha, M.Vaishnavi, J.Sasi Rabiyathul Sabena, A.P.L.Sangeetha, M.Santhi, "Realtime Monitoring Of Cardiac Patients At Distance Using Tarang Communication", International Journal of Innovative Research in Engineering \& Science (IJIRES), Volume 9, Issue 3,September 2014,pp-15-20

[4] Christo Ananth, G.Poncelina, M.Poolammal, S.Priyanka, M.Rakshana, Praghash.K., "GSM Based AMR", International Journal of Advanced Research in Biology, Ecology, Science and Technology (IJARBEST), Volume 1,Issue 4,July 2015, pp:26-28

[5] Christo Ananth, Kanthimathi, Krishnammal, Jeyabala, Jothi Monika, Muthu Veni, "GSM Based Automatic Electricity Billing System", International Journal Of Advanced Research Trends In Engineering And Technology (IJARTET), Volume 2, Issue 7, July 2015), pp:16-21

[6] Christo Ananth, G.Gayathri, M.Majitha Barvin, N.Juki Parsana, M.Parvin Banu, "Image Segmentation by Multi-shape GCOAAM", American Journal of Sustainable Cities and Society (AJSCS), Vol. 1, Issue 3, January 2014, pp 274-280

[7] Christo Ananth, D.L.Roshni Bai , K.Renuka, C.Savithra, A.Vidhya, "Interactive Automatic Hepatic Tumor CT Image Segmentation", International Journal of Emerging Research in Management \&Technology (IJERMT), Volume-3, Issue-1, January 2014,pp 16-20

[8]Christo Ananth, D.L.Roshni Bai, K.Renuka, A.Vidhya, C.Savithra, "Liver and Hepatic Tumor Segmentation in 3D CT Images", International Journal of Advanced Research in Computer Engineering \& Technology (IJARCET), Volume 3,Issue-2, February 2014,pp 496-503

[9] Christo Ananth, A.Sujitha Nandhini, A.Subha Shree, S.V.Ramyaa, J.Princess, "Fobe Algorithm for Video Processing", International Journal of Advanced Research in Electrical,
Electronics and Instrumentation Engineering (IJAREEIE), Vol. 3, Issue 3,March 2014 , pp 7569-7574

[10] Christo Ananth, Karthika.S, Shivangi Singh, Jennifer Christa.J, Gracelyn Ida.I, "Graph Cutting Tumor Images", International Journal of Advanced Research in Computer Science and Software Engineering (IJARCSSE), Volume 4, Issue 3, March 2014,pp 309-314

[11] Christo Ananth, G.Gayathri, I.Uma Sankari, A.Vidhya, P.Karthiga, "Automatic Image Segmentation method based on ALG", International Journal of Innovative Research in Computer and Communication Engineering (IJIRCCE), Vol. 2, Issue 4, April 2014,pp- 3716-3721

[12] Christo Ananth, A.S.Senthilkani, S.Kamala Gomathy, J.Arockia Renilda, G.Blesslin Jebitha, Sankari @Saranya.S., "Color Image Segmentation using IMOWT with 2D Histogram Grouping", International Journal of Computer Science and Mobile Computing (IJCSMC), Vol. 3, Issue. 5, May 2014, pp-1 - 7

[13] Christo Ananth, A.S.Senthilkani, Praghash.K, Chakka Raja.M., Jerrin John, I.Annadurai, "Overlap Wavelet Transform for Image Segmentation", International Journal of Electronics Communication and Computer Technology (IJECCT), Volume 4, Issue 3 (May 2014), pp-656-658

[14] Christo Ananth, S.Santhana Priya, S.Manisha, T.Ezhil Jothi, M.S.Ramasubhaeswari, "CLG for Automatic Image Segmentation", International Journal of Electrical and Electronics Research (IJEER), Vol. 2, Issue 3, Month: July - September 2014, pp: $51-57$

[15] Christo Ananth, R.Nikitha, C.K.Sankavi, H.Mehnaz, N.Rajalakshmi, "High Resolution Image Reconstruction with Smart Camera Network", International Journal of Advanced Research in Biology, Ecology, Science and Technology (IJARBEST), Volume 1,Issue 4,July 2015, pp:1-5

[16] Christo Ananth, B.Prem Kumar, M.Sai Suman, D.Paul Samuel, V.Pillai Vishal Vadivel, Praghash.K., "Autonomous Mobile Robot Navigation System", International Journal of Advanced Research in Biology, Ecology, Science and Technology (IJARBEST), Volume 1,Issue 4,July 2015,pp:15-19

[17] Christo Ananth , Mersi Jesintha.R., Jeba Roslin.R., Sahaya Nithya.S., Niveda V.C.Mani, Praghash.K., "Vision based Path Planning and Tracking control using Mobile Robot", International Journal of Advanced Research in Biology, Ecology, Science and Technology (IJARBEST), Volume 1,Issue 4,July 2015, pp:20-25

[18] Christo Ananth, S.Suryakala, I.V.Sushmitha Dani, I.Shibiya Sherlin, S.Sheba Monic, A.Sushma Thavakumari, "Vector Image Model to Object Boundary Detection in Noisy Images", International Journal of Advanced Research in Management, 
Architecture, Technology and Engineering (IJARMATE), Volume 1,Issue 2,September 2015, pp:13-15

[19] Christo Ananth," Geo-cutting Liver Tumor", International Journal of Advanced Research in Management, Architecture, Technology and Engineering (IJARMATE), Volume 2,Issue 3, March 2016,pp:122-128

[20] Christo Ananth, K.Kalaiselvi, C.Kavya, S.Selvakani, P.Sorimuthu Iyan, "Patterns for Next generation Database Systems - A study", International Journal of Advanced Research in Management, Architecture, Technology and Engineering (IJARMATE), Volume 2, Issue 4, April 2016, pp: 114-119

[21] Christo Ananth, S.Esakki Rajavel, S.Allwin Devaraj, M.Suresh Chinnathampy. "RF and Microwave Engineering (Microwave Engineering)." (2014): 300,ACES Publishers

[22] Christo Ananth, S.Esakki Rajavel, S.Allwin Devaraj, P.Kannan. "Electronic Devices." (2014): 300, ACES Publishers.

[23] Christo Ananth,W.Stalin Jacob,P.Jenifer Darling Rosita. "A Brief Outline On ELECTRONIC DEVICES \& CIRCUITS." (2016): 300 .

[24] Leonard G. C. Hamey, Colin Priest, "Automatic Number Plate Recognition for Australian Conditions", Proceedings of the Digital Imaging Computing: Techniques and Applications (DICTA), pp. 14- 21, December 2005.

[25] Yo-Ping Huang, Shi-Yong Lai,Wei-Po Chuang, "A Templateased Model for License Plate Recognition", IEEE International Conference on Networking, Sensing \& Control,March 21-23, 2004

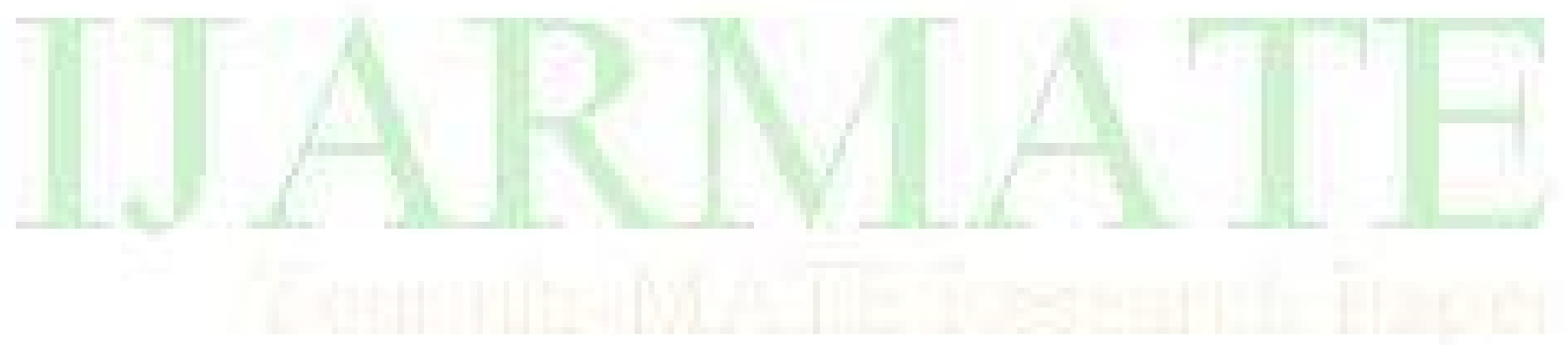

